\title{
An Inverse Optimal Control Approach for the Transfer of Human Walking Motions in Constrained Environment to Humanoid Robots
}

\author{
Debora Clever \\ Optimization in Robotics \& Biomechanics (ORB) \\ Interdisciplinary Center for Scientific Computing (IWR) \\ University of Heidelberg \\ debora.clever@iwr.uni-heidelberg.de
}

\author{
Katja Mombaur \\ Optimization in Robotics \& Biomechanics (ORB) \\ Interdisciplinary Center for Scientific Computing (IWR) \\ University of Heidelberg \\ katja.mombaur@iwr.uni-heidelberg.de
}

\begin{abstract}
In this paper we present an inverse optimal control based transfer of motions from human experiments to humanoid robots and apply it to walking in constrained environments. To this end we introduce a 3D template model, which describes motion on the basis of center of mass trajectory, foot trajectories, upper body orientation and phase duration. Despite of its abstract architecture with prismatic joints combined with damped series elastic actuators instead of knees, the model (including dynamics and constraints) is suitable to describe both, human and humanoid locomotion with appropriate parameters. We present and apply an inverse optimal control approach to identify optimality criteria based on human motion capture experiments. The identified optimal strategy is then transferred to the humanoid robot for gait generation by solving an optimal control problem, which takes into account the properties of the robot and differences in the environment. The results of this approach are the center of mass trajectory, the foot trajectories, the torso orientation, and the single and double support phase durations for a sequence of multiple steps allowing the humanoid robot to walk within a new environment. We present one computational cycle (from motion capture data to an optimized robot motion) for the example of walking over irregular step stones with the aim to transfer the motion to two very different humanoid robots (iCub Heidelberg01 and HRP-2 14). The transfer of these optimized robot motions to the real robots by means of inverse kinematics is work in progress and not part of this paper.
\end{abstract}

\section{INTRODUCTION}

Humanoid gait generation in constrained environments is a complex task which consists of a wide range of sub-tasks reaching from scene recognition [11, 31] and state estimation [27] over path planning [32] and pattern generation [17, 28] and finally to the computation of a whole body motion based on e.g. joint angles [21, 20]. To accomplish all those tasks, it is desirable to observe humans themselves and prolongate the gained knowledge to the robotic complements. As a specific sub-task of the before mentioned challenges, the focus of this paper is on the transfer of motion generation strategies from humans to humanoids. Previous work on this topic with focus on a direct transfer of joint angle trajectories has been published by [6], transfer based on observations in human gait on soft ground in [12]. Our approach goes further in both directions. In contrast to [6] it allows for the

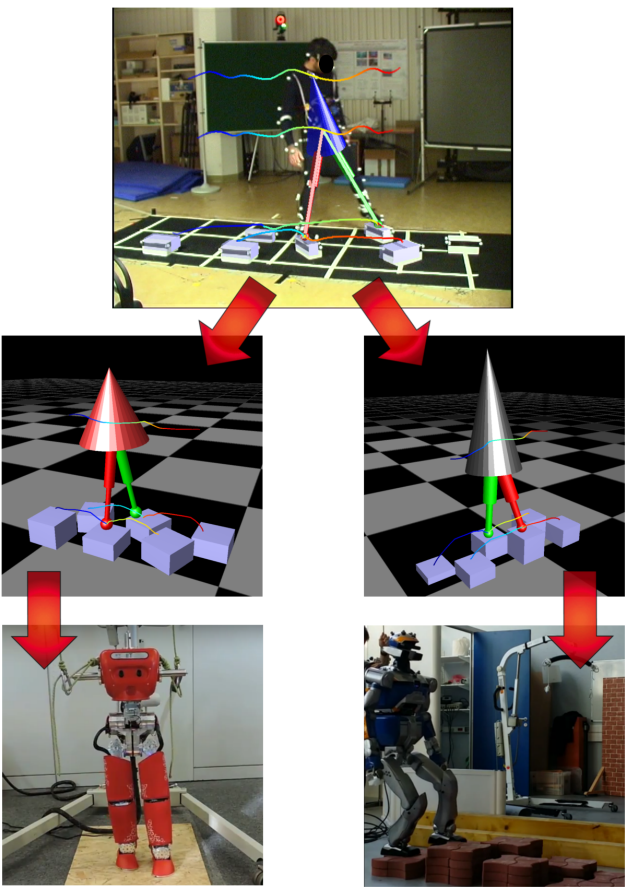

Fig. 1. Starting from real motion capture experiments, locomotion is transferred by means of identified optimality criteria for a dynamic template model (top) to the humanoid robot models (middle). In a subsequent step (not part of this paper) the motion is transferred to the real robots HeiCub and HRP-2 using inverse kinematics (bottom).

generation of robot motions without performing human motion experiments for this specific task. In contrast to [12] we use a model based approach to obtain a mathematical description of the observations made in human experiments. To this end we base our work on the hypothesis that the human motor system is optimal in its choice of motion [3, 4], use inverse optimal control and a human multi-body model to identify the underlying optimization criteria, adjust the optimal control model to the robots constraints and finally use optimal control, to compute a feasible robot motion based on the identified criteria (see Figures 12). 
To describe human and humanoid locomotion, in this work we consider the same 3D dynamical template model, adjusted to the specific embodiment of humans and robots by means of parameters and constraints. The model consists of two legs with prismatic joints combined with damped series elastic actuators instead of knees, two point-masses as feet and a reactive mass as upper body. Motion is described on basis of center of mass trajectory, feet trajectories, upper body orientation and phase duration [7].

Making use of template models for the identification of optimality criteria is an interesting approach for robotic applications for the following reasons:

- The same model can be used for different walking scenarios,

- The same model can be used for human gait analysis and humanoid gait generation,

- A sequence of several steps can be considered,

- Computational results are directly usable for robot controllers if they are based on the same template model,

- It has potential to be used for robot control in real time.

In this paper we present one entire computational cycle, starting from motion capture experiments and ending with the generation of humanoid motions based on center of mass trajectory, torso orientation, feet trajectories, and phase timing. As a proof of concept we focus on one trial of constrained walking and two humanoid robots. The first robot is the iCub platform of Heidelberg University (HeiCub), a unique version of iCub produced by iCub Facility Department of Fondazione Istituto Italiano di Tecnologia (IIT) [15, 24]. It has 15 degrees of freedom and consists of an upper body, a hip and two legs with a total height of $0.97 \mathrm{~m}$ and a total mass of $26 \mathrm{~kg}$. It does neither have arms nor a head and is therefore quite similar to the considered template model. The second robot is HRP-2 14 from Kawada, located at LAAS-CNRS [19, 18], a full body bi-pedal robot with 30 degrees of freedom. It has a total height of $1.54 \mathrm{~m}$ and a total mass of $58 \mathrm{~kg}$.

As constrained environment we consider a step stone scenario, which is particularity interesting but also difficult, because

- it combines several steps of different step length, direction and duration for which common optimality criteria have to be found,

- it includes constraints on the foot placement, and potential variation in ground height,

- it allows to consider significantly different scenarios for optimality identification and for motion generation.

Note, that the presented approach is usable for a wide range of different scenarios and can be used for the transfer to basically any bi-pedal robot. In parallel, but not part of this paper, we investigate how the identification of criteria transfers between different subjects and how optimality criteria correlate between different scenarios.

The paper is organized as follows: In Section two we explain the general idea of inverse optimal control and the solution strategy considered in this paper. In Section three we

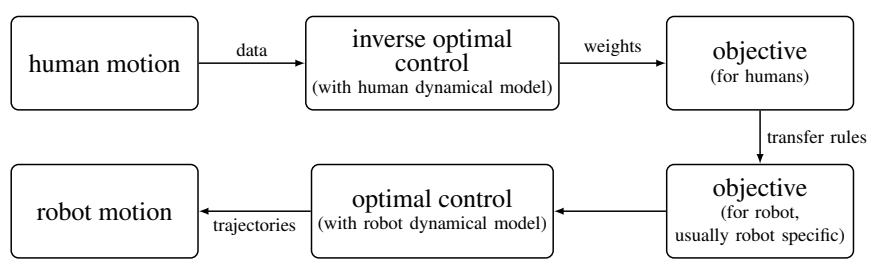

Fig. 2. The role of inverse optimal control for motion transfer between humans and humanoids based on optimality criteria.

present our 3D template model, which is suitable to mimic human and humanoid motion at the same time, by adjusting relevant parameters and constraints. In Section four we solve the inverse optimal control problem, based on the presented template model, a large set of optimality criteria for the optimal control model and real motion capture data of a step stone scenario. Finally, in Section five, the identified criteria together with suitable transfer rules, mainly based on speed issues and different bounds between human and humanoid, are used to compute optimal motions for the two different humanoid robot models each walking over a different step stone scenario.

\section{InVERSE Optimal CONTROL FOR HUMAN GAIT ANALYSIS AND HUMANOID GAIT GENERATION}

The overall goal of our research and the EU-project KoroiBot is to improve humanoid walking capabilities by learning from humans. In this context inverse optimal control serves as a tool to extract optimization criteria from human gait and derive humanoid locomotion by minimizing these criteria with respect to the robot's architecture and bounds (see Figure 2).

\section{A. Problem formulation}

Based on the assumption that human gait is optimal [3, 4] with respect to well defined but unknown criteria, we describe human gait by a mathematical model in the form of an optimal control problem restricted by a system of differential algebraic equations (DAEs) and additional non-linear constraints on control and state.

Given a reference motion $\hat{\mathbf{Z}}=\left(\hat{z}_{i, t_{j}}\right)_{i=0, \ldots, N_{z}-1, j=0, \ldots, N_{\hat{t}}-1}$ describing $N_{z}$ trajectories at discrete times $\left(\hat{t}_{0}, \hat{t}_{1}, \ldots, \hat{t}_{N_{\hat{t}}-1}\right)$, we use inverse optimal control to determine an objective function $\Phi^{*}(\mathbf{x}, \mathbf{u}, \mathbf{q}, \mathbf{p}, \gamma)$ such that the subset $\mathbf{z}(t) \subset$ $(\mathbf{x}(t), \mathbf{u}(t), \mathbf{q})$ of the resulting optimal solution $\left(\mathbf{x}^{*}, \mathbf{u}^{*}, \mathbf{q}^{*}\right)$ approximates $\hat{\mathbf{Z}}$ (after scaling it with $\boldsymbol{\omega} \in \mathbb{R}^{N_{z}}$ ) as well as possible.

$$
\begin{aligned}
& \min _{(\mathbf{p}, \boldsymbol{\gamma})} \frac{1}{N_{z} N_{\hat{t}}} \sum_{i=0}^{N_{z}-1} \omega_{i} \sum_{j=0}^{N_{\hat{t}}-1}\left(z_{i}\left(t_{j}\right)-\hat{z}_{i, t_{j}}\right)^{2}, \\
& \text { s.t. } \min _{(\mathbf{x}, \mathbf{u}, \tilde{\mathbf{q}})} \Phi(\mathbf{x}, \mathbf{u}, \tilde{\mathbf{q}}, \mathbf{p}, \boldsymbol{\gamma}) \\
& \text { s.t. } \dot{\mathbf{x}}=\mathbf{f}_{k}(\mathbf{x}, \mathbf{u}, \mathbf{q}, \mathbf{p}), k=0, \ldots, n_{s}-1 \\
& \quad \mathbf{r}_{k}^{(e q)}(\mathbf{x}, \mathbf{u}, \mathbf{q}, \mathbf{p})=0, \mathbf{r}_{k}^{(i n e q)}(\mathbf{x}, \mathbf{u}, \mathbf{q}, \mathbf{p}) \geq 0, \\
& \quad \mathbf{c}_{k}\left(\mathbf{x}\left(\tau_{0}\right), \ldots, \mathbf{x}\left(\tau_{n_{s}}\right), \mathbf{q}, \mathbf{p}\right) \geq 0, \\
& \text { and } \quad \gamma_{0}=\gamma_{0}^{(0)} .
\end{aligned}
$$


The lower level (2)-(5) describes the optimal control problem of minimizing the objective function $\Phi(\mathbf{x}, \mathbf{u}, \mathbf{q}, \mathbf{p})$ with respect to the walking model (3)-(5), the upper level (1) is a parameter estimation problem for the unknown weights $\gamma \in$ $\mathbb{R}^{N_{\gamma}}:=\left(\gamma_{i}\right)_{i=0, \ldots, N_{\gamma}-1}$ and optionally some of the parameters $\mathbf{p} \in \mathbb{R}^{N_{p}}$, such that the optimized motion $\mathbf{z}^{*} \subset\left(\mathbf{x}^{*}, \mathbf{u}^{*}, \mathbf{q}^{*}\right)$ is fitted to real motion capture data $\hat{\mathbf{Z}}$. Equation (6) belongs to the upper level problem and ensures uniqueness of the parameter set $\gamma$ by fixing $\gamma_{0}$ to its initial value. The state $\mathbf{x}:\left[t_{0}, t_{f}\right] \rightarrow \mathbb{R}^{N_{x}}$ and the control $\mathbf{u}:\left[t_{0}, t_{f}\right] \rightarrow \mathbb{R}^{N_{u}}$ are vector functions in time, the control parameters $\mathbf{q} \in \mathbb{R}^{N_{q}}$ and the model parameters $\mathbf{p} \in \mathbb{R}^{N_{p}}$ are vectors of real numbers. The right hand side $\mathbf{f}:=\left(f_{0}, \ldots, f_{n_{s}-1}\right)$ is phase-wise defined (denoted by the subscript $k$ ) and allows for discontinuities on phase boundaries. Switching condition between phases are defined implicitly in the context of the constraints $\mathbf{c}_{k}$, which also cover other point-wise constraints like periodicity requirements. As the right hand side, the path constraints $\mathbf{r}_{k}$ are defined phase-wise.

One important issue in the analysis of human gait are studies on the duration of the different phases of the gait cycle - or even more challenging: studies on optimality criteria leading to realistic phase durations. To this end, the durations $\Delta \boldsymbol{\tau}$ between the implicit defined switching points $\left(\tau_{0}, \ldots, \tau_{n_{s}}\right)$ have to be included in the vector of control parameters $\tilde{\mathbf{q}}:=$ $[\mathbf{q}, \Delta \boldsymbol{\tau}]$. Note, that there is a important difference between the parameters $\mathbf{q}$ (and $\tilde{\mathbf{q}}$, respectively) and $\mathbf{p}$. Whereas $\mathbf{q}$ is a control parameter of the lower problem, $\mathbf{p}$ is a model parameter, and hence fixed for the lower problem. However, it can be considered as an identification parameter on the upper level.

Usually the reference trajectories $\hat{\mathbf{Z}}$ do neither include the control nor do they define the entire state, but only a subset of it. A special feature of the presented algorithm is that it can handle arbitrary subsets $\mathbf{z}$, since it does not build on the assumption that for the reference motion all state and control trajectories are explicitly given. It can even handle the special case, if $\hat{\mathbf{Z}}$ does not correspond to a subset $\mathbf{z}(t) \subset$ $(\mathbf{x}(t), \mathbf{u}(t), \mathbf{q})$ but to a general function $\mathbf{M}(\mathbf{x}(t), \mathbf{u}(t), \mathbf{q})$.

In our research we define the lower objective function of the optimal control model (2)-(5) as a linear combination $\Phi(\mathbf{x}, \mathbf{u}, \mathbf{q}, \mathbf{p}, \boldsymbol{\gamma}):=\sum_{i=0}^{N_{\gamma}-1} \gamma_{i} \phi_{i}(\mathbf{x}, \mathbf{u}, \mathbf{q}, \mathbf{p})$, of physically meaningful sub-criteria $\phi_{i}, i=0, \ldots, N_{\gamma}-1$ which can describe common criteria known from biomechanics or robotics or new hypotheses based on human experiments. There are two major advantages to use this kind of objective function: first, using physically meaningful sub-criteria, the results of gait analysis are physically interpretable and second, it implies the possibility to derive transfer rules from humans to humanoids which can take into account dynamic and kinematic differences between the two embodiments.

\section{B. Solution strategy}

To solve the inverse optimal control problem (17) - (6) there are to two main types of solution strategies. In the first strategy the bi-level structure of the problem is kept and exploited [25,
26, 29]. In the second one the bi-level problem is replaced by a one-level problem, substituting the lower level problem by its optimality conditions [8, 9, 14, 5, 13, 2]. In our research we consider and also compare both approaches. In this paper we focus on the first approach which has shown to perform well for real-life optimal control problems and allows for an easy implementation of new problems. An one-level approach for similar studies, but with an easier template model walking on level ground, has been considered in complementary work.

As both, the upper and the lower level of the bi-level optimal control problem are optimization problems themselves, we need suitable solution strategies. Whereas the upper level is a parameter identification problem with constraints of unusual form, the lower level problem is restricted by a hybrid differential equation with additional linear and nonlinear constraints, where known structures can be exploited. Therefore on the upper level we rely on a derivative free optimization method, in this paper the quadratic approximation based method BOBYQA [30], implemented in the optimization library NLOPT [16]. For the lower level, we use a derivative based approach which relies on a state parametrization by multiple shooting and a structure exploiting SQP algorithm to solve the resulting discrete nonlinear programs (NLPs). Both algorithms have been combined in an efficient inverse optimal control framework that allows the user

- to define an optimal control model, which consists of an objective function defined by a linear combination of optimization criteria, a dynamical model given by a hybrid differential equation, and additional linear and nonlinear constraints (also specifying implicit phase changes),

- to define the sub-set of optimization parameters to be taken into account on the upper level, which usually consists of the objective weights $\gamma$ and a subset of model parameters $\mathbf{p}$,

- to define the sub-set of quantities which are the ones that are fitted to motion capture data or functions thereof. These quantities are usually a subset of the state $\mathbf{x}$, and the subset $\Delta \boldsymbol{\tau} \subset \mathbf{q}$, defining the duration of the different phases.

\section{Locomotion as Optimal Control Model}

As mentioned above, in our work we model locomotion as an optimal control problem, relying on the hypothesis that the human motor control system is optimal in its choice of motions. For our framework, such an optimal control model consists of three main parts. First, the dynamic multi-body model, which we usually model on bases of Newtons law, reformulating the differential algebraic equations of index three as a system index one. Second, the gait cycle as a sequence of changing contact sets and additional linear and non-linear constraints. Different dynamical behavior as a consequence of varying contact sets during the gait cycle is defined by different phases, resulting in a hybrid differential equation. Phase durations are defined implicitly by characteristic state and control dependent switching events. Furthermore, we explicitly allow for discontinuities in the state functions 
to model the impact of foot touch down. Third, a twice continuously differentiable objective function. As stated above, in the context of inverse optimal control, we formulate the objective as a linear combination of different criteria. Note, that the criteria themselves can be highly non-linear.

\section{A. The dynamical walking model and the step stones}

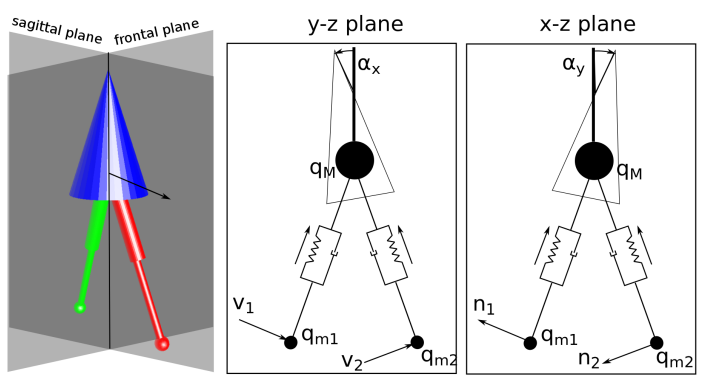

Fig. 3. 3D SEA-walker

We consider the three dimensional dynamical template model (3D SEA-walker), which we have first introduced in [7], and slightly tailor it to the arising needs. It consists of an upper body (torso) and two legs, which are attached to the center of mass of the torso. Both legs have a compliant knee, modeled by a damped series elastic actuator, and a point foot with appropriate mass.

Denoting the absolute positions of the center of mass of the torso and feet positions by

$$
\mathbf{q}_{M}:=\left(\begin{array}{c}
x_{M} \\
y_{M} \\
z_{M}
\end{array}\right), \mathbf{q}_{m 1}:=\left(\begin{array}{c}
x_{m 1} \\
y_{m 1} \\
z_{m 1}
\end{array}\right), \mathbf{q}_{m 2}:=\left(\begin{array}{c}
x_{m 2} \\
y_{m 2} \\
z_{m 2}
\end{array}\right),
$$

and defining the torso mass $M$, the feet masses $m_{1}$ and $m_{2}$, the leg length of the $i$-th leg $l_{i}$, and the inertia tensor of the torso $\Theta$, the dynamics of the motion is defined by the following equations:

$$
\begin{aligned}
M \ddot{\mathbf{q}}_{M} & =\frac{F_{1}}{l_{1}}\left(\mathbf{q}_{M}-\mathbf{q}_{m 1}\right)+\frac{F_{2}}{l_{2}}\left(\mathbf{q}_{M}-\mathbf{q}_{m 2}\right)-M \mathbf{g} \\
m_{1} \ddot{\mathbf{q}}_{m 1} & =\mathrm{RHS}_{\mathrm{m} 1}, m_{2} \ddot{\mathbf{q}}_{m 2}=\mathrm{RHS}_{\mathrm{m} 2} \\
\boldsymbol{\Theta} \ddot{\boldsymbol{\alpha}} & =\mathbf{u}_{\tau 1}+\mathbf{u}_{\tau 2} .
\end{aligned}
$$

We set

$$
\boldsymbol{\alpha}:=\left(\begin{array}{c}
\alpha_{x} \\
\alpha_{y}
\end{array}\right), \mathbf{u}_{\tau 1}:=\left(\begin{array}{l}
u_{x 1} \\
u_{y 1}
\end{array}\right), \mathbf{u}_{\tau 2}:=\left(\begin{array}{l}
u_{x 2} \\
u_{y 2}
\end{array}\right), \mathbf{g}:=\left(\begin{array}{l}
0 \\
0 \\
g
\end{array}\right),
$$

with gravitational acceleration $g$. Including a series elastic actuator (SEA) and a damper, the leg forces are given as

$$
F_{i}=-k\left(l_{i}-l_{0}-u_{s i}\right)-b \cdot i_{i},
$$

where $k$ is the spring constant, $b$ the damping constant, $l_{i}$ the leg length, $l_{0}$ the rest length, $\mathbf{u}_{s}=\left(u_{s 1}, u_{s 2}\right)^{T}$ the control of the SEA, $\mathbf{u}_{\tau}=\left(\mathbf{u}_{\tau 1}, \mathbf{u}_{\tau 2}\right)^{T}$ the hip torque, and $\boldsymbol{\alpha}$ the torso orientation. Each step is modeled as a sequence of single support phase (SS), instantaneous touch down discontinuity

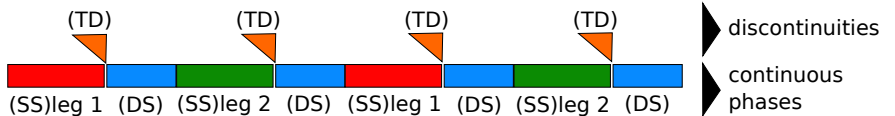

Fig. 4. Sequence of walking phases.

(TD), and double support phase (DS), see Figure 4. In our computations, we consider a sequence of two full strides, where each stride contains two steps with alternating legs. During the different phases of one single step, we formulate the following right hand sides and additional constraints:

- Single support phase (SS) on leg $i$, with swing foot $j$

$$
\begin{aligned}
\text { RHS }_{\mathrm{mi}} & =0, \\
\text { RHS }_{\mathrm{mj}} & =-\frac{F_{j}}{l_{j}}\left(\mathbf{q}_{M}-\mathbf{q}_{m j}\right)+\frac{u_{x j}}{l_{j}} \mathbf{v}_{j}+\frac{u_{y j}}{l_{j}} \mathbf{n}_{j}-m_{j} \mathbf{g}, \\
l_{j} & \leq l_{0}+u_{s j}, \quad z_{m j} \geq h\left(x_{m j}, y_{m j}\right),
\end{aligned}
$$

- Discontinuity at touch down (TD), leg $j$

$$
\begin{aligned}
& \dot{\mathbf{q}}_{m j}=0, \quad\left(x_{m j}, y_{m j}\right) \in C_{\text {stepstones }}, \\
& z_{m j}=h\left(x_{m j}, y_{m j}\right)
\end{aligned}
$$

- Transition conditions (SS, leg $i) \rightarrow(\mathrm{DS}$, leg $j$ in front)

$$
z_{m j}=h\left(x_{m j}, y_{m j}\right), \quad \dot{z}_{m j} \leq 0
$$

- Double support phase (DS, leg $j$ in front)

$$
\begin{aligned}
\mathrm{RHS}_{\mathrm{mi}} & =0, \quad \mathrm{RHS}_{\mathrm{mj}}=0, \\
l_{i} & \leq l_{0}+u_{s i}, \quad l_{j} \leq l_{0}+u_{s j},
\end{aligned}
$$

- Transition conditions (DS, leg $j$ in front $) \rightarrow($ SS, leg $j$ )

$$
\operatorname{grf}_{z, i}=0, \quad i_{i} \geq c>0, \quad l_{j} \leq l_{0}+u_{s j},
$$

where $\operatorname{grf} f_{z, i}$ is the vertical ground reaction force of leg $i$.

Furthermore we fix $\left(x_{M}, y_{M}\right)$ at initial time $t=0$ and final time $T$ to a desired value, to allow to use the same model for later gait generation. The subsequent step is defined analogously to (11)-(19) by switching the roles of leg $i$ and $j$.

Different from the version of the SEA-walker in [7], as upper body we consider a regular cone, with center of mass $(\mathrm{CoM})$ at $\frac{h_{t}}{4}$ and inertia tensor $\Theta=\frac{M}{80} \operatorname{diag}\left(12 r_{t}^{2}+3 h_{t}^{2}, 12 r_{T}^{2}+\right.$ $3 h_{t}^{2}, 24 h_{t}^{2}$ ) (cone radius $r_{t}$, cone height $h_{t}$ ). Furthermore we consider the torso orientation $\boldsymbol{\alpha}=\left(\alpha_{x}, \alpha_{y}\right)$ around the global $x$ and $y$ axis, in analogy to the absolute positions of CoM and feet. The vector $\mathbf{v}_{i}$ lies in th $y-z$ plane and is perpendicular to the vector from $\mathbf{q}_{m i}$ to $\mathbf{q}_{M}$, the vector $\mathbf{n}_{i}$ is lies in the $x-z$ plane and is also perpendicular to the vector from $\mathbf{q}_{m i}$ to $\mathbf{q}_{M}$. The torque $u_{x i}$ implies a linear force in direction of $v_{i}$ on foot $i$, the torque $u_{y i}$ implies a linear force in direction of $n_{i}$ on foot $i$. The function $h: \mathbb{R}^{2} \rightarrow \mathbb{R}$ defines the height of the uneven ground, the set $C_{\text {stepstones }}$ the feasible region to be stepped on. 
We parameterize the $n_{s}$ step stones by $N_{s}=4 \cdot 3 \cdot\left(n_{s}\right)$ parameters $p_{i}$, which correspond to the markers placed on the corners of each step stone during the experiments. Therefore, for each considered motion, the step stone model is automatically adjusted to position, orientation and dimension of the real blocks. Feasible $(x, y)$-regions are described by the four lines, passing through the $(x, y)$ coordinates of two adjacent markers, with its normal pointing inwards. A foot placement is defined to be feasible, if the distance between the $(x, y)$-position of the foot and all four lines is greater than a safety margin and if the position $(x, y, z)$ fulfills the condition $h(x, y)=z$, with function $h$ describing the height of the ground or the step stones at a point $(x, y)$.

\section{B. Optimality criteria}

In this work, we propose a set of twelve different optimality criteria $\phi_{i}, i=0, \ldots, 11$, depending on state $\mathbf{x}$, control $\mathbf{u}$, and one optimality parameter $p_{D S S S}$ which defines the optimal ratio of double and single support. Note, that all objective functions of Lagrange type are additionally divided by the corresponding phase duration $\Delta \tau_{k}$ to avoid an implicit minimization of phase times. As described above, the objective function to be identified is defined as follows

$$
\Phi\left(\mathbf{x}, \mathbf{u}, p_{D S S S}\right)=\sum_{i=0}^{11} \gamma_{i} \phi_{i}(\cdot),
$$

with the optimality functions of Lagrange type: minimization of the SEA actuation in the stance foot

$$
\begin{aligned}
\phi_{0}(\mathbf{u}) & =\min \int_{\mathrm{SS} 1} u_{s 1}^{2} \mathrm{~d} t \\
& +\min \int_{\mathrm{SS} 2} u_{s 2}^{2} \mathrm{~d} t+\min \int_{\mathrm{DS}} u_{s 1}^{2}+u_{s 2}^{2} \mathrm{~d} t,
\end{aligned}
$$

minimization of the SEA actuation in the swing foot

$$
\phi_{1}(\mathbf{u})=\min \int_{\mathrm{SS} 1} u_{s 2}^{2} \mathrm{~d} t+\min \int_{\mathrm{SS} 2} u_{s 1}^{2} \mathrm{~d} t,
$$

minimization of hip torque of the swing foot

$$
\phi_{2}(\mathbf{u})=\min \int_{\mathrm{SS} 1}\left\|\mathbf{u}_{\tau 2}\right\|^{2} \mathrm{~d} t+\min \int_{\mathrm{SS} 2}\left\|\mathbf{u}_{\tau 1}\right\|^{2} \mathrm{~d} t,
$$

minimization of angular momentum in $x$-direction

$$
\phi_{3}(\mathbf{x})=\min \int_{0}^{T}\left(\Theta_{x} \cdot \dot{\alpha}_{x}\right)^{2} \mathrm{~d} t,
$$

minimization of angular momentum in $y$-direction

$$
\phi_{4}(\mathbf{x})=\min \int_{0}^{T}\left(\Theta_{y} \cdot \dot{\alpha}_{y}\right)^{2} \mathrm{~d} t,
$$

minimization of vertical center of mass oscillations

$$
\phi_{5}(\mathbf{x})=\min \int_{0}^{T} \dot{z}_{M}^{2} \mathrm{~d} t,
$$

minimization of absolute swing foot velocity

$$
\phi_{6}(\mathbf{x})=\min \int_{S S 1}\left\|\dot{\mathbf{q}}_{m 2}\right\|^{2} \mathrm{~d} t+\min \int_{S S 2}\left\|\dot{\mathbf{q}}_{m 1}\right\|^{2} \mathrm{~d} t,
$$

and the optimality functions of Mayer type minimization of planar distance between foot position at touch down and capture point

$$
\begin{aligned}
\phi_{7}(\mathbf{x}) & =\sum_{\text {all steps }} \min \left(\left\|\left(x_{m 1}\left(t_{t d 1}\right), y_{m 1}\left(t_{t d 1}\right)\right)^{T}-\boldsymbol{\xi}\left(t_{t d 1}\right)\right\|\right. \\
& \left.+\left\|\left(x_{m 2}\left(t_{t d 2}\right), y_{m 2}\left(t_{t d 2}\right)\right)^{T}-\boldsymbol{\xi}\left(t_{t d 2}\right)\right\|\right) \\
\boldsymbol{\xi}(t) & =\left(x_{M}(t), y_{M}(t)\right)^{T}+\sqrt{\frac{l_{0}}{g}}\left(\dot{x}_{M}(t), \dot{y}_{M}(t)\right)^{T}
\end{aligned}
$$

minimization of periodicity gap in center of mass velocities

$$
\phi_{8}(\mathbf{x})=\min \left\|\dot{\mathbf{q}}_{M}(0)-\dot{\mathbf{q}}_{M}(T)\right\|^{2}
$$

minimization/maximization of overall single support duration

$$
\phi_{9}(\mathbf{x})= \pm \sum_{\text {all steps }}\left(q_{t, s s 1}+q_{t, s s 2}\right),
$$

minimization of absolute swing foot velocity at touch down

$$
\phi_{10}(\mathbf{x})=\sum_{\text {all steps }} \min \left(\left\|\dot{\mathbf{q}}_{m 1}\left(t_{t d 1}\right)\right\|^{2}+\left\|\dot{\mathbf{q}}_{m 2}\left(t_{t d 2}\right)\right\|^{2}\right),
$$

tracking of ratio between sub-sequent double and single support phase to constant but unknown value $p_{D S S S}$

$$
\phi_{11}(\mathbf{x})=\sum_{\text {all steps }}\left\|\frac{q_{t, d s}}{q_{t, s s}}-p_{D S S S}\right\|^{2} .
$$

\section{OPTIMALITY CRITERIA IDENTIFICATION}

For the identification of optimality criteria, or to be more precise, of the optimality weights $\gamma$ and optimality criteria related parameters $\mathbf{p}$, we rely on a least square fit of model $\mathrm{CoM}$, feet trajectories, torso orientation, and phase durations to the corresponding reference trajectories and reference times.

In accordance to common notation in optimal control of ODEs and DAEs and with respect to Section II] in the following we refer to the control as $\mathbf{u}:=\left(\mathbf{u}_{s}, \mathbf{u}_{\tau}\right)$ and to the state as $\mathbf{x}:=\left(\mathbf{q}_{M}, \mathbf{q}_{m 1}, \mathbf{q}_{m 2}, \boldsymbol{\alpha}, \dot{\mathbf{q}}_{M}, \dot{\mathbf{q}}_{m 1}, \dot{\mathbf{q}}_{m 2}, \dot{\boldsymbol{\alpha}}\right)$. However, $x$ as a subscript denotes the $x$-axis of the basis frame.

\section{A. Model parameters and reference data}

As in the first part of the presented motion transfer, the 3D-SEA walker is meant to mimic a human being, the model parameters have to be set as close as possible to the corresponding values of the subject and the environment of the motion capture experiment.

Environment and subject specific data (such as block size and location, leg length, torso height, etc.) can directly be measured. Feet and upper body masses are approximated based on de Leva [10] and the absolute weight of the subject. The width of the cone is set to $r_{t}:=\frac{1}{2} h_{t}$. Spring constant $k$ and damping factor $b$ are chosen by use of optimal control, such that for a minimized amount of actuation the model mimics the main characteristics of human walking. All relevant parameters are given in Table $\mathbb{I}$

We use motion capture and subject specific data, jointly recorded with M. Giese et al. at Eberhard Karls University, 
TABLE I

WALKING MODEL PARAMETERS AND BOUNDS FOR HUMAN SUBJECT

\begin{tabular}{l|c} 
& human subject \\
\hline body weight & $60 \mathrm{~kg}$ \\
est. upper body weight & $30 \mathrm{~kg}$ \\
foot weight & $0.3 \mathrm{~kg}$ \\
leg rest length & $0.965 \mathrm{~m}$ \\
torso height & $0.7 \mathrm{~m}$ \\
torso radius & $0.35 \mathrm{~m}$ \\
spring stiffness & $3300 \mathrm{~kg} / \mathrm{s}^{2}$ \\
damping constant & $660 \mathrm{Ms} / \mathrm{m}$ \\
bound ss duration $(1, \mathrm{u})$ & $0.4 \mathrm{~s}, 0.6 \mathrm{~s}$ \\
bound ds duration $(1, \mathrm{u})$ & $0.2 \mathrm{~s}, 0.5 \mathrm{~s}$ \\
safety margin on block & $(0.02,0.02,0.02,0.02) \mathrm{m}$ \\
(front, left, back, right) & \\
\hline
\end{tabular}

Tübingen, and published in the open source KoroiBot motion database, set up by Karlsruhe Institute of Technology [23, 1].

To convert the recorded motion to model related reference data we extract a mean hip point $\mathbf{q}_{M, r e f}=\frac{1}{4}(L A S I * R A S I *$ $L H I P * R H I P)$, mean foot points $\left(x_{m, r e f}, y_{m, r e f}\right)=$ $\frac{1}{2}(T O E+H E E L), z_{m, r e f}=\min (T O E, H E E L)$, and the angles $\alpha_{x}$ and $\alpha_{y}$ given by the orientation of a line through the mean hip point $\mathbf{q}_{M \text {, ref }}$ and the marker C7. LASI, RASI, LHIP, RHIP, TOE, HEEL and C7 are marker labels and denote markers for which three dimensional position trajectories are recorded. For a full description of the used marker set (and its labeling) we refer to the marker set, introduced in [22].

The reference motion starts, when the rear leg is about to lift off from the floor while the front leg is already on the first tread. The motion ends after four full steps, when the front leg is on the last step stone and the rear leg is about to take off again.

\section{B. Identification of objective weights}

To take into account the different orders of magnitude of the twelve optimality functions 21-32, we introduce a scaling vector $\boldsymbol{\sigma}$, which ensures that the elements of the weight vector $\tilde{\gamma}:=\left(\gamma^{T}, p_{D S S S} / \sigma_{D S S S}\right)^{T}$ are of similar magnitude, to enforce a well conditioned upper optimization problem. Here, we set $\sigma$ to

$$
\boldsymbol{\sigma}=\{1,1,0.01,10,10,1,0.01,0.1,0.1,0.001,0.001,0.1,0.25\},
$$

and the initial weights to

$$
\tilde{\gamma}^{(0)}=\{1.0,1.0,1.0,1.0,1.0,1.0,1.0,1.0,1.0,0.5,1.0,1.0,1.0\} .
$$

For uniqueness reasons, we fix the first weight $\tilde{\gamma}_{0}$ to one and propose an initial ratio for double and single support of 1:4 $\left(p_{D S S S}=0.25\right)$. Since the identification of $p_{D S S S}$ would be infeasible for the case $\tilde{\gamma}_{11}=0$ or $\tilde{\gamma}_{12}=0$, we introduce the bounds $0.1 \leq \tilde{\gamma}_{11} \leq 10,0.01 \leq \tilde{\gamma}_{12} \leq 10$. Furthermore, to allow for both, minimization and maximization of the overall single support duration, we define the bounds $-10 \leq \tilde{\gamma}_{9} \leq 10$. For all other weighs we require non-negativity $0 \leq \tilde{\gamma}_{i} \leq 10$, $i=0, \ldots, 10, i \neq 9$.

For the initial guess of $\tilde{\gamma}^{(0)}$ we observe an average quadratic deviation between motion capture data and computed optimal motion of $0.04 \mathrm{~m}$ and an average difference in phase times of 0.11 s. After 121 iterations on the upper level the algorithm converges with

$\tilde{\gamma}^{*} \approx\{1.0,1.3,0.8,0.92,0.93,0.91,0.91,1.35,1.28,0.51,0.98,0.1,1.61\}$.

For the identified weights the average quadratic deviation of the optimized motion can be reduced to $0.03 \mathrm{~m}$ and $0.06 \mathrm{~s}$.

A comparison between a representative subset of the computed quantities to their reference counterpart is presented in Figure 5, a comparison of phase durations in Figure 6
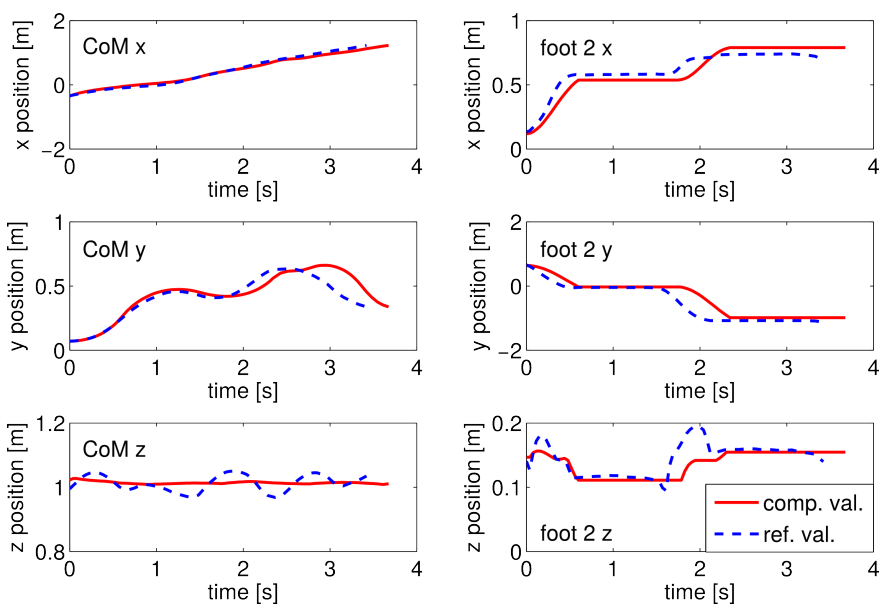

Fig. 5. CoM trajectory and left foot trajectory resulting from identified objective function (red, solid line) versus marker based reference data (blue, dashed line). The $x$-direction is the walking direction.

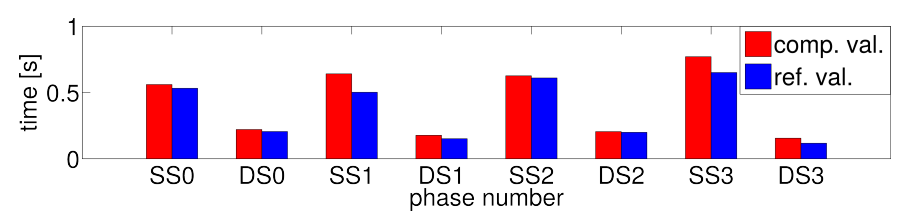

Fig. 6. Phase durations resulting from identified objective function (red) versus reference data (blue).

Phase durations, feet trajectories, and $\mathrm{x}-\mathrm{y}$ coordinates of the center of mass are satisfying reconstructed. The torso orientation and the vertical CoM velocities (Figure 5, bottom left) have a greater relative difference, which is due to their small scaling and the fact that the upper level fitting is based on absolute errors. This can be changed by adjusting the weight vector $\omega$, see Equ. (1).

The optimal motion for the identified objective function, together with indicators of the reference motion, is given in the figure sequence 7

\section{Humanoid Gait Generation}

Comparing the two humanoid robots to the human model we can observe significant differences in architecture and dynamical behavior.

The first robot, the iCub platform of Heidelberg University (HeiCub), has 15 degrees of freedom and consists of an upper body, a hip and two legs [15, 24]. It does neither have arms nor 

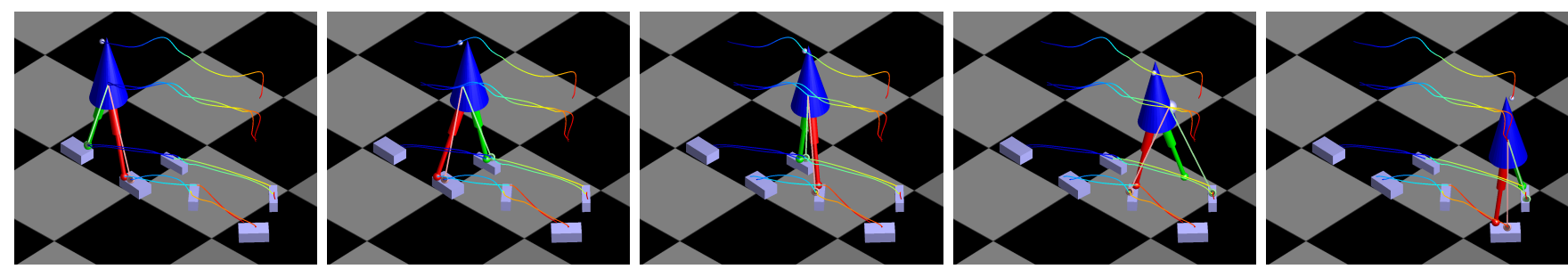

Fig. 7. Optimal motion with respect to the identified weights. The motion of interest is shown by the 3D-SEA walker. The reference motion is sketched by light colored lines as legs and a small ball as head. The head trajectory of the motion (which is not an explicit state) is only plotted for the reference solution and is meant to simplify the comparison of the torso orientation. A corresponding video is available online: http://orb.iwr.uniheidelberg.de/ftp/CleverMombaur_IOC_RSS2016

a head and is therefore quite similar to the considered template model. It has a total height of $0.97 \mathrm{~m}$ and a total mass of $26 \mathrm{~kg}$. The second robot, HRP-2, is a full body bi-pedal robot with 30 degrees of freedom. It has a total height of $1.54 \mathrm{~m}$ and a total mass of $58 \mathrm{~kg}$ [19, 18].

Most of the differences between the three embodiments (human model, HeiCub (red), HRP-2 (gray)) can be directly taken into account by adjusting the model parameters, model constraints and environment constraints. Whereas we have precise information about robot masses and limits, we have no information about spring and damping constants in terms of the considered SEA-model. In the following experiments we use the same stiffness as for the human but reduce the damping to $440 \mathrm{Ms} / \mathrm{m}$. The relevant parameters and limits are given in Table II

TABLE II

PARAMETERS AND BOUNDS FOR ROBOT MODELS

\begin{tabular}{l|cc} 
& HeiCub (red) & HRP-2 (gray) \\
\hline body weight & $26 \mathrm{~kg}$ & $58 \mathrm{~kg}$ \\
est. upper body weight & $12.5 \mathrm{~kg}$ & $28 \mathrm{~kg}$ \\
foot weight & $0.8 \mathrm{~kg}$ & $1.2 \mathrm{~kg}$ \\
leg rest length & $0.6 \mathrm{~m}$ & $0.75 \mathrm{~m}$ \\
torso height & $0.38 \mathrm{~m}$ & $0.8 \mathrm{~m}$ \\
torso radius & $0.38 \mathrm{~m}$ & $0.4 \mathrm{~m}$ \\
spring stiffness & $3300 \mathrm{~kg} / \mathrm{s}^{2}$ & $3300 \mathrm{~kg} / \mathrm{s}^{2}$ \\
damping constant & $440 \mathrm{Ms} / \mathrm{m}$ & $440 \mathrm{Ms} / \mathrm{m}$ \\
bound ss duration $(1, \mathrm{u})$ & $0.5 \mathrm{~s}, 1.5 \mathrm{~s}$ & $0.5 \mathrm{~s}, 1.5 \mathrm{~s}$ \\
bound ds duration $(1, \mathrm{u})$ & $0.15 \mathrm{~s}, 0.5 \mathrm{~s}$ & $0.15 \mathrm{~s}, 0.5 \mathrm{~s}$ \\
safety margin to edge & $(0.10,0.05, \ldots$ & $(0.12,0.08, \ldots$ \\
(front, left, back, right) & $\ldots, 0.05,0.05) \mathrm{m}$ & $\ldots, 0.08,0.08) \mathrm{m}$ \\
\hline
\end{tabular}

We set up two different step stone scenarios, one for each robot. Here, the second scenario is particularity interesting as it has already been accomplished by HRP-2 14 at CNRS-LAAS, using a standard pattern generator.

\section{A. Transfer rules}

Performing motion transfer based on optimality, as it is done in this paper, it is desirable to transfer the identified weights of human optimality criteria as direct as possible to the humanoid objective function. However, as robots are much more constrained than humans a direct transfer of objective weights usually drives the robot model to some of its bounds. Those active constraints can change the nature of the motion dramatically. Therefore, we aim to identify transfer rules in terms of weight re-scaling which prevent the model to be stuck on a certain bound.

In our specific example (as most likely for many other ones) the identified weight for the minimization of single support phase duration is much too large for the robot and would result in phase durations which are forced to its lower bound. However, this would mean the same as predefining phase durations and destroys the advantage of state dependent phase transitions. Therefore, it is reasonable to not only adjust the lower bounds for the phase duration, but also to decrease the weight $\tilde{\gamma}_{9}$. Similar observations are made if we only include stricter bounds on the swing foot velocity. Here, an active bound results in an undesired M-shaped swing foot trajectory. Therefore, in addition to the stricter bound the weight $\tilde{\gamma}_{6}$ has to be increased. For the first robot model (HeiCub) the weight $\tilde{\gamma}_{9}$ is divided by two $\left(\tilde{\gamma}_{9, r o b 1}=0.25\right)$, which is the only modification we introduce for this robot. For the second robot model (HRP-2), where we define componentwise bounds on the CoM velocity of $[-0.5,0.5] \mathrm{m} / \mathrm{s}$ and on the swing foot velocities of $[-0.8,0.8] \mathrm{m} / \mathrm{s}$, we increase $\tilde{\gamma}_{6}$ to $\tilde{\gamma}_{6, \text { rob } 2}=2$ and set $\tilde{\gamma}_{9}$ to the high negative value of $\tilde{\gamma}_{9, \text { rob } 2}=-6$. Furthermore, we include the fact that in previous experiments (using a standard pattern generator) HRP-2 successfully crossed the considered step stone scenario with a ratio of double to single support of $1 / 7$. Hence, for the HRP-2 model we set the parameter $p_{D S S S, r o b 2}=0.14$. Also modifications in environment constraints might require a rescaling of optimality parameters. In our example, HRP-2 is asked to manage steps with significantly greater differences in step height than in the training step stone scenario. Because the actuation of the SEA also mimics desired knee bending, setting $\gamma_{1}=1.3$ enforces a gait, where the robot model swings its almost stretched legs outwards instead of swinging the foot on a more direct line between step stones and adjusting the leg length. To this end we divide this weight by two and set $\gamma_{1, \text { rob } 2}=0.65$. In scenario 1 the topology of the step stones is quite similar to the human example, except for the smaller distances and the fact, that the robot has to start the motion with the other leg. Due to the characteristics of the template model this issue does not require any additional modifications, neither in the dynamics of the model, nor in the constraints, nor in the objective. 


\section{B. Gait synthesis}
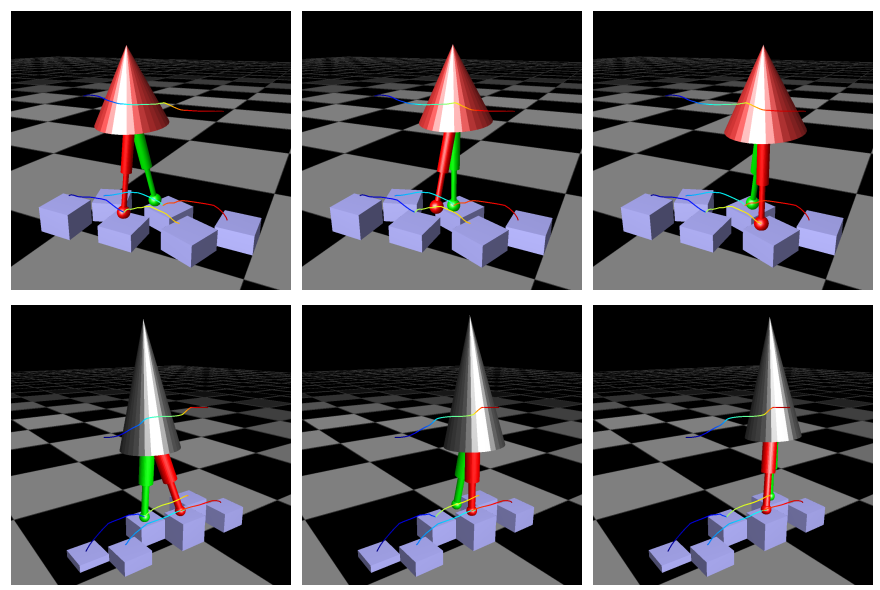

Fig. 8. Robot motions as result of an optimal control problem in terms of CoM and feet trajectories, torso orientation and phase durations. Objective weights, model parameters and bounds are adjusted to the constraints of the two robots. A corresponding video is available online: http://orb.iwr.uniheidelberg.de/ftp/CleverMombaur_IOC_RSS2016

We now use the same optimal control model which we have used in the lower level of the inverse optimal control approach, but adjust parameter and objective scaling as described in Table $\Pi$ and Subsection $\mathrm{V}-\mathrm{A}$. As we do not have a sophisticated guess for an initial motion (as we had for the inverse optimal control based on the motion capture experiments) we start with a simple initial motion, where feet and CoM trajectories are linearly interpolates between step postures and where the torso orientation is constant to zero. Finally, we predefine the desired values for the initial horizontal CoM position $\left(x_{M}, y_{M}\right)$ at time $t=0$ and the final ones at time $T$.
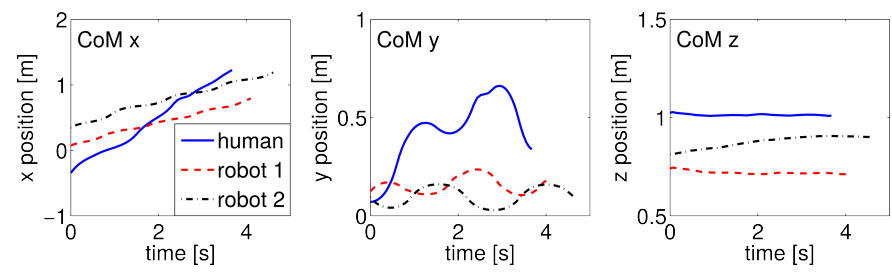

Fig. 9. CoM trajectories resulting from identified objective function for the human model (blue) and the two robot models, HeiCub (red) and HRP-2 (black).

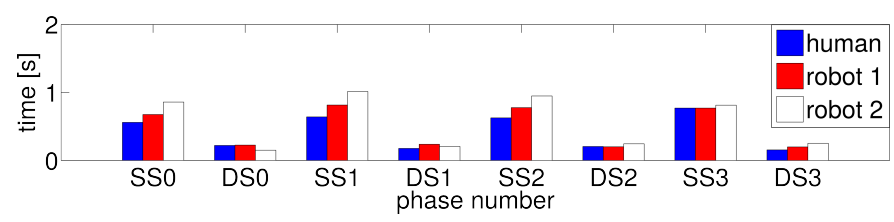

Fig. 10. Phase durations resulting from identified objective function for the human model (blue) and the two robot models, HeiCub (red) and HRP-2 (white).

The result of this computation are optimal trajectories for the center of mass, the feet, the torso angles and the phase durations for the two robot models, see Figure 8 . Exemplary for all the quantities, the CoM trajectories of the human motion and the humanoid motions are presented in Figure 9 Whereas the human motion is quite fast $\left(v_{\varnothing}=0.43 \mathrm{~m} / \mathrm{s}\right)$, the computed motion for the robots, with the reduced weight for time minimization, results in much slower motions (both $v_{\varnothing}=0.18 \mathrm{~m} / \mathrm{s}$ ), see Figure 9 and 10

\section{Conclusions}

On basis of a step stone scenario, we have presented an approach, which allows to transfer walking motions in constrained environment from humans to humanoids that have different embodiments and are moving within a new scenario with significantly different properties.

Even though the optimal motion resulting from the identified optimization strategy does not exactly match the one based on motion capture markers, the inverse optimal control algorithm shows a satisfying performance. The average error in trajectories and timing between the computed and the reference motion is reduced by $40 \%$ in comparison to the best hand tuned solution. Taking 121 upper level iterations for full convergence, already after half of the iterations, suitable weights are identified. More precisely, this means that for a loss of accuracy by approximately $5 \%$ computational time can be reduced by $50 \%$. We have shown, that the optimal control based transfer of motion leads to reasonable robot motions for two different robots. We can observe the advantage of physically interpretable optimization criteria to derive heuristic (but physically motivated) transfer rules. An important step of future research is a systematic investigation and formulation of similar transfer rules which hold for a wide range of scenarios and robots. Follow up work will focus on two issues. On the computational side, we extend of our studies to different walking scenarios and augment the optimality identification across different trials and different subjects. On the practical side, we investigate on how well the computed robot motions transfer to their real counterparts. In this context, the computed motions are not planned to be run open loop on the robot. Rather they substitute existing methods (e.g. based on the table cart model) to generate the input for the robot specific whole body motion generation tools.

\section{ACKNOWLEDGMENTS}

The research leading to these results has received funding from the EU 7th Framework Program (FP7/2007-2013) under grant agreement no 611909 (KoroiBot). We thank the Simulation and Optimization group of H. G. Bock at the University of Heidelberg for providing the optimal control code Muscod-II. Furthermore we thank our KoroiBot partners: M. Giese (CIN Tübingen) and team for performing the motion capture experiments, T. Asfour (H2T, KIT) and team for maintaining the motion capture database, and P. Souères and O. Stasse (CNRS-LAAS) for their support towards the transfer of concept to the real robot HRP-2. 


\section{REFERENCES}

[1] KoroiBot Motion Capture Database. https://koroibotmotion-database.humanoids.kit.edu/. Last visited April 2016.

[2] S. Albrecht, P. Basili, S. Glasauer, M. Leibold, and M. Ulbrich. Modeling and analysis of human navigation with crossing interferer using inverse optimal control In Proceedings of the Vienna International Conference on Mathematical Modeling, 2012.

[3] R. M. Alexander. The gaits of bipedal and quadrupedal animals The International Journal of Robotics Research, 3(2):49-59, 1984.

[4] R. M. Alexander. Optima for animals. Princeton University Press, 1996.

[5] G. B. Allende and G. Still. Solving bilevel programs with the KKT-approach. Mathematical programming, 138(12):309-332, 2013.

[6] L. Boutin, A. Eon, S. Zeghloul, and P. Lacouture. From human motion capture to humanoid locomotion imitation application to the robots HRP-2 and HOAP-3. Robotica, 29(02):325-334, 2011.

[7] D. Clever and K. Mombaur. A new template model for optimization studies of human walking on different terrains. In Humanoid Robots (Humanoids), 2014 14th IEEE-RAS International Conference on, pages 500-505. IEEE, 2014.

[8] D. Clever and K. Mombaur. On the relevance of common humanoid gait generation strategies in human locomotion - an inverse optimal control approach. In Hoang X.P. Rannacher R. Schlöder J. Bock, H.G., editor, Modeling, Simulation and Optimization of Complex Processes HPSC 2015 (to appear). Springer, 2016.

[9] D. Clever, K. Hatz, and K. Mombaur. Studying Dynamical Principles of Human Locomotion using Inverse Optimal Control. In PAMM, volume 14, pages 801-802. Wiley Online Library, 2014.

[10] P. De Leva. Adjustments to Zatsiorsky-Seluyanov's segment inertia parameters. Journal of biomechanics, 29 (9):1223-1230, 1996.

[11] D. Gonzalez-Aguirre, M. Vollert, T. Asfour, and R. Dillmann. Robust Real-time 6D Active-visual Localization for Humanoid Robots In IEEE International Conference on Robotics and Automation (ICRA 2014), pages 27852791, 2014.

[12] K. Hashimoto, H. Kang, M. Nakamura, E. Falotico, H. Lim, A. Takanishi, C. Laschi, P. Dario, and A. Berthoz. Realization of biped walking on soft ground with stabilization control based on gait analysis. In Intelligent Robots and Systems (IROS), 2012 IEEE/RSJ International Conference on, pages 2064-2069. IEEE, 2012.

[13] K. Hatz. Efficient numerical methods for hierarchical dynamic optimization with application to cerebral palsy gait modeling. $\mathrm{PhD}$ thesis, University Heidelberg, 2014.

[14] K. Hatz, J. P. Schlöder, and H. G. Bock. Estimating
Parameters in Optimal Control Problems. SIAM Journal on Scientific Computing, 34(3):A1707-A1728, 2012.

[15] Y. Hu, K. Mombaur, and F. Nori. Using optimal control to generate squat motions for the humanoid robot iCub with SEA. Proceedings of Dynamic Walking, 2015.

[16] S. G. Johnson. The NLopt nonlinear-optimization package. http://ab-initio.mit.edu/nlopt, 2014.

[17] S. Kajita, F. Kanehiro, K. Kaneko, K. Fujiwara, K. Yokoi, and $\mathrm{H}$. Hirukawa. A realtime pattern generator for biped walking In Robotics and Automation, 2002. Proceedings. ICRA'O2. IEEE International Conference on, volume 1, pages 31-37. IEEE, 2002.

[18] S. Kajita, T. Nagasaki, K. Kaneko, K. Yokoi, and K. Tanie. A running controller of humanoid biped HRP2LR. 2005.

[19] K. Kaneko, F. Kanehiro, S. Kajita, K. Yokoyama, K. Akachi, T. Kawasaki, S. Ota, and T. Isozumi. Design of prototype humanoid robotics platform for HRP. In Intelligent Robots and Systems, 2002. IEEE/RSJ International Conference on, volume 3, pages 2431-2436. IEEE, 2002.

[20] A Koenemann, A. Del Prete, Y. Tassa, E. Todorov, O. Stasse, M. Bennewitz, and N. Mansard. Experiments with MuJoCo on HRP-2 In IEEE/RSJ International Conference on Intelligent Robots and Systems (IROS 2015), 2015.

[21] M. Kudruss, M. Naveau, O. Stasse, N. Mansard, C. Kirches, P. Souères, and K. Mombaur. Optimal Control for Whole-Body Motion Generation using Centerof-Mass Dynamics for Predefined Multi-Contact Configurations. In IEEE/RAS International Conference on Humanoid Robots (Humanoids 2015), page 7, November 2015.

[22] C. Mandery, Ö. Terlemez, N. Vahrenkamp, and T. Asfour. The Koroibot Markerset. Technical report, Institute for Anthropomatics and Robotics, Karlsruhe Institute for Technology (KIT), Karlsruhe, Germany, 2014.

[23] C. Mandery, Ö. Terlemez, M. Do, N. Vahrenkamp, and T. Asfour. The KIT Whole-Body Human Motion Database. In IEEE International Conference on Advanced Robotics (ICAR 2015), pages 329-336, 2015.

[24] G. Metta, L. Natale, F. Nori, G. Sandini, D. Vernon, L. Fadiga, C. Von Hofsten, K. Rosander, M. Lopes, J. Santos-Victor, et al. The iCub humanoid robot: An open-systems platform for research in cognitive development. Neural Networks, 23(8):1125-1134, 2010.

[25] K. Mombaur, H. G. Bock, and J. P. Schlöder. Open-loop stable solutions of periodic optimal control problems in robotics ZAMM - Journal of Applied Mathematics and Mechanics, 85(7):499-515, 2005.

[26] K. Mombaur, A. Truong, and J.-P. Laumond. From Human to Humanoid Locomotion An inverse optimal control approach. Autonomous Robots, 28(3), 2010.

[27] F. Nori, N. Kuppuswamy, and S. Traversaro. Simultaneous state and dynamics estimation in articulated structures In IEEE/RSJ International Conference on 
Intelligent Robots and Systems (IROS 2015), pages 33803386, 2015.

[28] A. Pajon, G. De Magistris, S. Miossec, K. Kaneko, and A. Kheddar. A humanoid walking pattern generator for sole design optimization. In IEEE International Conference on Advanced Robotics (ICAR 2015), pages 105-110, 2015.

[29] T. Park and S. Levine. Inverse optimal control for humanoid locomotion. In Robotics Science and SystemsWorkshop on Inverse Optimal Control and Robotic Learning from Demonstration, 2013.

[30] M. J. D. Powell. The BOBYQA algorithm for bound constrained optimization without derivatives. 2009.

[31] O. Ramos, M. Garcia, N. Mansard, O. Stasse, J. B. Hayet, and P. Souères. Towards reactive vision-guided walking on rough terrain: an inverse dynamic based approach. International Journal of Humanoid Robotics (IJHR), 11: 1004-1026, 2014.

[32] M. Sreenivasa, K. Mombaur, and J. P. Laumond. Walking paths to and from a goal differ: On the role of bearing angle in the formation of human locomotion paths. Plos one, 10(4), 2015. 